\title{
Preface for the special issue devoted to AISC 2014
}

\author{
Jacques Calmet ${ }^{1}$
}

Published online: 12 April 2017

(C) Springer International Publishing Switzerland 2017

This special issue is devoted to selected and improved papers presented at the 12th International Conference on Artificial Intelligence and Symbolic Computation (AISC 2014). This conference was organized by the University of Sevilla in Spain and held during December 11-13, 2014 in the Department of Computer Sciences and Artificial Intelligence at the University of Sevilla.

AISC 2014 was the latest in the series of specialized biennial conferences founded in 1992 by Jacques Calmet and John Campbell. Its goal was to investigate original research contributions in the fields of artificial intelligence (AI) and symbolic computation: At the origin of the series, the conferences were called AISMC where the letter "M" stood for mathematical. From 1998 on, the scope became broader than only mathematics and the "M" was dropped. This trend lead to associate the conference to potentially similar ones, including Calculemus and Mathematical Knowledge Management (MKM), under the integrated federation of Conference on Intelligent Computer Mathematics (CICM).

From the beginning, the proceedings have appeared as volumes in the LNCS and then the LNAI series. Several special issues have also appeared over the years.

AISC 2014 became again independent from the CICM federation in order to extend the scope to new domains covered by AI and symbolic computation beyond classical mathematics. The goals were, on the one hand, to bind mathematical domains such as algebraic topology or algebraic geometry to AI, but also, on the other hand, to link AI to domains outside pure algorithmic computing.

Thus, the new scope covers domains which can be described generically as belonging to the mechanization and computability of artificial intelligence. This includes the basic concepts of computability and Turing machines, new logics including non-classical ones, reasoning, learning, decision support systems, but also machine intelligence and

Jacques Calmet

jfcalmet@yahoo.fr

Karlsruhe Institute of Technology (KIT), Institute for Theoretical Informatics,

Köslinerstr. 1876139 Karlsruhe, Germany 
epistemology and philosophy of symbolic mathematical computing. Theoretical as well as application papers were solicited.

Among the 22 submitted papers, 16 were selected for publication in LNAI 8884 edited by Gonzalo A. Aranda-Corral, Jacques Calmet and Francisco J. Martín-Mateos. Of those, five papers were expanded and improved to be published in this special issue of AMAI after undergoing journal refereeing. While not fully covering the expected new scope outlined for this series, the special issue illustrates nicely the trend being set for these conferences.

The first paper, Computing envelopes in dynamic geometry environments by Francisco Botana and Tomas Recio, is set in the domain of automated deduction in geometry. After reviewing the behavior of some popular dynamic geometry software when computing envelopes, special attention is given to the new GeoGebra 5.0 version. This system incorporates a mathematically rigorous approach for envelope computations.

The next paper, Conformant planning as a case study of incremental QBF solving by Uwe Egly, Martin Kronegger, Florian Lonsing and Andreas Pfandler, provides results for the first empirical study of incremental QBF (Quantified Boolean Formulas) solving in the context of planning with uncertainty. The authors then motivate how to use similar methodologies in other application domains. The methodology can be outlined through the following keywords: conformant planning, incremental solving, preprocessing and blocked clause elimination.

The third paper, Design and implementation of maple packages for processing offsets and conchoids by Juana Sendra, David Gómez Sánchez-Pascuala and Valerio Morán, is very similar to usual applications of computer algebra systems, but, in fact, the domain of application is not that usual. It describes two packages, implemented in Maple, for dealing with offsets and conchoids to algebraic curves, respectively. They are applied to two rational families of algebraic plane curves providing an useful atlas. A feature is that the computing times remain fully reasonable.

In cognitive science, conceptual blending has been proposed as a mechanism to facilitate the creation of new concepts and ideas by constrained combination of available knowledge. The fourth paper, Theory blending: extended algorithmic aspects and examples by M. Martinez, A. M. H. Abdel-Fattah, U. Krumnack, D. Gómez-Ramírez, A. Smaill, T. R. Besold, A. Pease, M. Schmidt, M. Guhe and K.-U. Kühnberger, investigates extended algorithmic aspects of theory blending. It describes a logic-based framework which allows a formal treatment particularly suitable for applications in mathematics. The methodology implemented deals with heuristic-driven theory projection.

The last paper, Local and global symmetry breaking in itemset mining by Belaïd Benhamou, is concerned with local and global symmetry breaking in item-set mining. The concept of symmetry is extensively used in constraint programming and propositional satisfiability. It can be exported to the area of data mining where some tasks can be expressed as constraints or logical formulas. This work investigates the notion of local symmetries and compares it to global symmetry for the data mining of item-set problems. 